\title{
Endothelin-1 Regulation Is Entangled in a Complex Web of Epigenetic Mechanisms in Diabetes
}

\author{
S. BISWAS ${ }^{1}$, B. FENG ${ }^{1}$, A. THOMAS ${ }^{1}$, S. CHEN ${ }^{1}$, E. AREF-ESHGHI ${ }^{1}$, B. SADIKOVIC ${ }^{1}$, \\ S. CHAKRABARTI ${ }^{1}$
}

${ }^{1}$ Department of Pathology and Laboratory Medicine, Western University, London, Ontario, Canada

Received December 12, 2017

Accepted February 15, 2018

\section{Summary}

Endothelial cells (ECs) are primary targets of glucose-induced tissue damage. As a result of hyperglycemia, endothelin-1 (ET-1) is upregulated in organs affected by chronic diabetic complications. The objective of the present study was to identify novel transcriptional mechanisms that influence ET-1 regulation in diabetes. We carried out the investigation in microvascular ECS using multiple approaches. ECs were incubated with $5 \mathrm{mM}$ glucose (NG) or $25 \mathrm{mM}$ glucose (HG) and analyses for DNA methylation, histone methylation, or long non-coding RNA- mediated regulation of ET-1 mRNA were then performed. DNA methylation array analyses demonstrated the presence of hypomethylation in the proximal promoter and $5^{\prime}$ UTR/first exon regions of EDN1 following $\mathrm{HG}$ culture. Further, globally blocking DNA methylation or histone methylation significantly increased ET-1 mRNA expressions in both NG and HG-treated HRECs. While, knocking down the pathogenetic IncRNAs ANRIL, MALAT1, and ZFAS1 subsequently prevented the glucose-induced upregulation of ET-1 transcripts. Based on our past and present findings, we present a novel paradigm that reveals a complex web of epigenetic mechanisms regulating glucose-induced transcription of ET-1. Improving our understanding of such processes may lead to better targeted therapies.

\section{Key words}

ET-1 - Epigenetics - IncRNAs - DNA methylation - Histone methylation

\section{Corresponding author}

S. Chakrabarti, Department of Pathology and Laboratory Medicine, Western University, London, Ontario, N6A 5C1, Canada. Dental Sciences Building 4033. Fax: (519) 663-2930. E-mail: subrata.chakrabarti@lhsc.on.ca

\section{Introduction}

With the continuous global rise in diabetes (IDF 2015), the risk for developing glucose-induced injuries to the microvasculature also remains high. As a consequence of hyperglycemia, microvascular damage gives rise to debilitating complications affecting the eyes, kidneys, and peripheral nerves (Fowler 2011). To minimize the risk of subsequent diabetes-induced vessel damage, current therapeutic modalities are comprised of lifestyle changes and pharmaceutical intervention (Chawla et al. 2016, Fowler 2011). Although these therapies may be effective in impeding some progression of late stage diabetic complications, the presence of 'metabolic memory' contributes to unique molecular alterations that may alter an individual's response to treatments (Roberto et al. 2017). Epigenetic mechanisms comprise the 'metabolic memory' phenomenon and identification of these mechanisms would expand current therapeutic modalities, allowing for the development of targeted treatment strategies.

Modifications to the epigenome influence gene expression without changing the underlying nucleotide sequence (Weinhold 2006). DNA methylation, histone modifications, and the activity of non-coding RNAs are examples of such epigenetic mechanisms that are involved in metabolic memory (Biswas and Chakrabarti 2017). The identification of these processes have been made evident by high throughput genomic technologies, however new questions arise with respect to our current understanding of the documented mechanistic properties for pro-inflammatory markers.

Endothelin-1 (ET-1) is a prominent peptide in 
diabetes that has been well documented within the last three decades. As previously demonstrated by our laboratory and others, aberrations in metabolic pathways can heighten the production of ET-1 and subsequently allow for an increased pathologic state in various diabetic complications (Chen et al. 2000, Chakrabarti et al. 1998, Takahashi et al. 1990). Using such knowledge and information from ligand-binding studies, drugs were developed to target the ET-1 receptors, in order to mitigate cellular dysfunction and other effects in diabetic complications (Sasser et al. 2007, Kohan and Pollock 2013). Despite the promising results for endothelin receptor antagonists in animal models, investigators discovered that there were adverse, undesirable and heterogeneous responses present among numerous patients using these drugs, suggesting that there are alternative mechanisms implicated in ET-1 regulation (Remuzzi et al. 2002). As the mechanistic impact of epigenetic machinery on ET-1 production still remains unclear, further characterization is warranted to understand such a process.

In this study, we examined the roles of DNA methylation, histone methylation, and long non-coding RNAs (lncRNAs) on ET-1 regulation in retinal microvascular endothelial cells (HRECs). We performed a DNA methylation array to identify whether unique methylation patterns exist across the ET-1 gene (EDN1) after high glucose (HG) incubation. To further follow-up our findings from the methylation array, we employed a global DNA methylation inhibitor in HRECs to determine the impact of DNA methyltransferases on ET-1 regulation. Next, we subjected HRECs to a histone methylation inhibitor to understand the influence of histone methyltransferases on ET-1 mRNA production. Moreover, based on our previous lncRNA microarray analysis (Thomas et al. 2017), we selected three prominent HG-induced upregulated IncRNAs and silenced these lncRNAs to determine the effects on ET-1. We then collated our previous and current findings to present an all-inclusive epigenetic paradigm for ET-1 regulation.

\section{Materials and Methods}

\section{Cell culture}

Endothelial dysfunction is one of the earliest pathological features during chronic hyperglycemia (Alder et al. 1997). Hence, we used HRECs (Olaf Pharmaceuticals; MA, USA) for our experiments. Such experimental conditions have been previously described (Puthanveetil et al. 2015, Thomas et al. 2017, Ruiz et al.
2015, Khan et al. 2004) and all cell culture reagents were purchased from Sigma (Oakville, ON, CAN). Based on our previous findings, the 48 -h time point was selected for our in vitro experiments. All experiments were independently repeated at least three times and performed with six replicates, unless specified.

\section{EDN1 CpG DNA methylation analysis}

The Illumina Infinium MethylationEPIC BeadChip array (Illumina; CA, USA) was used to identify differential methylation patterns of $\mathrm{CpG}$ sites across the EDN1 gene in HRECs incubated in $5 \mathrm{mM}$ glucose (NG, mimicking euglycemia) and in $25 \mathrm{mM}$ glucose (HG, mimicking hyperglycemia). Genomic DNA was extracted from HRECs after $48 \mathrm{~h}$ of glucose culture and $1 \mu \mathrm{g}$ of DNA was used for bisulfite conversion using the EZ DNA Methylation Kit (Zymo Research; CA, USA). The HiScan System (Illumina; CA, USA) was used to obtain the array readout and the methylated and unmethylated signal intensity data were then imported into R 3.4.0 for analysis. Normalization was performed using Illumina normalization method with background correction using the minfi package. Probes with detection $P$-value $>0.01$ were excluded from the downstream analyses. In addition, probes known to contain SNPs at the $\mathrm{CpG}$ interrogation or the single nucleotide extension were removed. Methylation level for each probe was measured as a beta value ( $\beta$-value), calculated from the ratio of the methylated signals versus the total sum of unmethylated and methylated signals, ranging between 0 (no methylation) and 1 (full methylation). Three independent samples were used per group.

\section{3-Deazaneplanocin A (DZNep) and 5-Aza-2'-deoxycytidine $(5-a z a-d C)$}

In order to understand the impact of histone and DNA methylation inhibition on ET-1, we used pharmacological inhibitors that globally blocked the methylation process. Therefore, based on previous literature, $1 \mathrm{~h}$ pre-treatment of either $5 \mu \mathrm{M}$ of DZNep (Cayman Chemical; MI, USA) or 5-aza-dC (Sigma; STL, USA) was applied to HRECs prior to addition of D-glucose (Ruiz et al. 2015, Thomas et al. 2017, Xie et al. 2014). DZNep or 5-aza-dC-treated HRECs, and their respective controls, were collected at $48 \mathrm{~h}$ for further analyses.

\section{SiRNA-mediated transfection}

HRECs were initially transfected with either two 
pre-designed siRNAs targeting human ANRIL (antisense RNA to INK4 locus), MALAT1 (metastasis-associated lung adenocarcinoma transcript 1), or ZFAS1 (zinc finger antisense 1), or scrambled siRNA (catalog number: AM4635, Life Technologies; CA, USA) using Lipofectamine 2000 (Invitrogen; ON, CA) and Opti-MEM (Life Technologies; CA, USA). Details regarding the lipofectamine-mediated transfection protocol can be found in our previous studies (Ruiz et al. 2015, Thomas et al. 2017). Following confirmation of knockdown by RT-qPCR, the siRNA sequences with the best knockdown were selected for this study: ANRIL (Lincode CDKN2B-AS1; catalog number: R-188105-000005, Dharmacon; IL, USA), MALAT1 (catalog number: n272233, Life Technologies; CA, USA), and ZFAS1 (catalog number: n271357, Life Technologies; CA, USA).

\section{Enzyme-Linked Immunosorbent Assay (ELISA)}

ET-1 protein levels were determined from cell supernatants of DZNep or 5-aza-dC-treated HRECs using the ET-1 Quantikine ELISA kit (R\&D Systems; MP, USA). Concentrations were first quantified using the BCA protein assay kit (Pierce; IL, USA) and $100 \mu \mathrm{g}$ of protein was equally used for each sample according to the manufacturer's instructions. The Multiskan FC Microplate Photometer (Thermo Fisher Scientific; MA, USA) determined the optical density for each well at $450 \mathrm{~nm}$ and corrected using $568 \mathrm{~nm}$.

RNA isolation and quantitative Real-Time Polymerase Chain Reaction (RT-qPCR)

As previously described, the TRIzol reagent (Invitrogen; Burlington, ON, CAN) was used to extract total RNA and a spectrophotometer $(260 \mathrm{~nm}$; Gene Quant, Pharmacia Biotech, USA) was utilized to quantify RNA concentrations (Ruiz et al. 2015, Thomas et al. 2017, Khan et al. 2004, Puthanveetil et al. 2015). A high-capacity complementary DNA (cDNA) kit (Applied Biosystems; Burlington, ON, CAN) was then employed to reverse transcribe $1 \mu \mathrm{g}$ of total RNA to cDNA. Next, in combination with SYBR-green master mix (Clontech, CA, USA) and specific target gene primers (Sigma; Table 1), cDNA was amplified in the LightCycler 96 System (Roche Diagnostics, QC, CA) to detect RNA expression. Expression levels were further calculated by the relative standard curve method using $\beta$-actin as internal control for normalization.

Table 1. Oligonucleotide sequences used for RT-qPCR.

\begin{tabular}{ll} 
Target gene & Oligonucleotide sequence $\left(\mathbf{5}^{\prime} \rightarrow \mathbf{3}^{\prime}\right)$ \\
\hline$\beta$-actin $($ Homo $)$ & Forward: CCTCTATGCCAACACAGTGC \\
& Reverse: CATCGTACTCCTGCTTGCTG \\
Endothelin-1 (Homo) & Forward: AAGCCCTCCAGAGAGCGTTAT \\
& Reverse: CCGAAGGTCTGTCTGTCACCAATGT
\end{tabular}

\section{Statistical analysis}

All statistical tests were performed using GraphPad Prism 7 (GraphPad, CA, USA). Data are expressed as mean \pm SEM. Student's $t$-test or 1-way ANOVA, followed by Tukey's post hoc test, were used as appropriate. Differences with a $P$ value below 0.05 were considered significant.

\section{Results}

Transient $H G$ treatment results in hypomethylation of $C p G$ sites in the proximal promoter regions of EDN1

Following our methylation profiling of NG and HG-treated HRECs, we exclusively selected the CpG sites that spanned across $E D N 1$, which corresponded to
12 probes (Supplementary File S1). All of the probes were placed in the 'Open Sea' category, since the array did not detect a $\mathrm{CpG}$ island (defined as regions that have a GC content greater than $50 \%$ and are greater than 200 base pairs in length; Gardiner-Garden and Frommer 1987 ) in the EDN1 region, which was also confirmed by the UCSC Human Genome Browser (University of California, Santa Cruz, CA, USA; Fig. 1A). Using annotations derived by Illumina, we further categorized the probes based on functional location relative to the gene region: TSS 1500 (region that is -200 to $-1,500$ nucleotides upstream from the transcription start site), TSS200 (the region from -200 nucleotides upstream to the transcription start site itself), 5' UTR (can include the region through the first exon), gene body, and 3' UTR. 


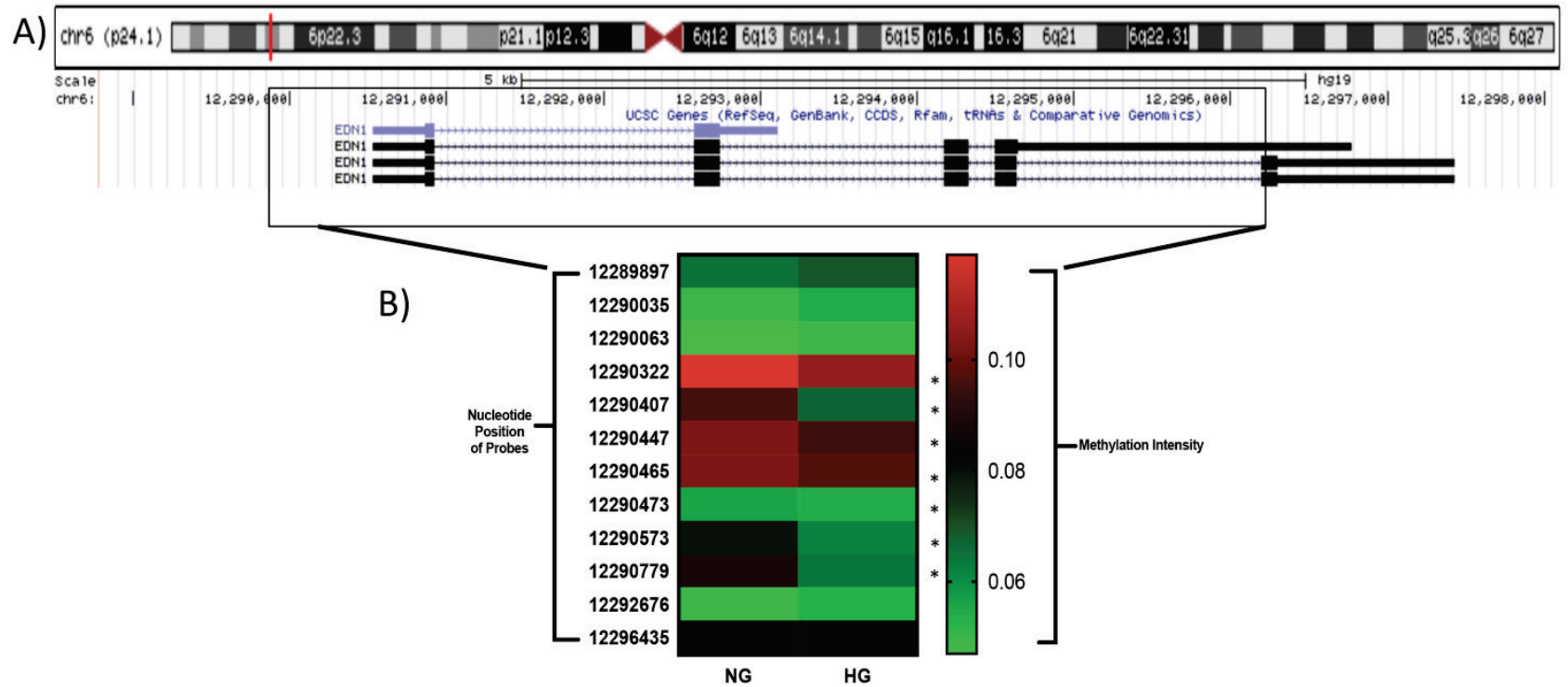

C)

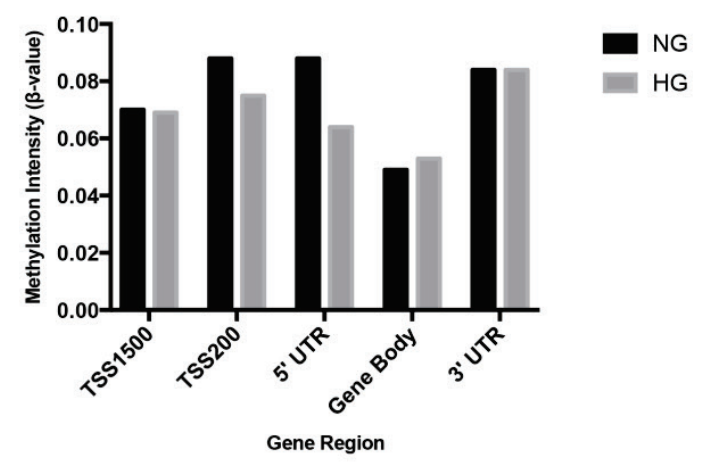

Fig. 1. $\mathrm{CpG}$ sites in specific regions of $E D N 1$ are hypomethylated following high glucose treatment in vitro. A) Information on the human $E D N 1$ gene annotated from the UCSC database. EDN1 is located on chromosome 6 and spans from the nucleotide positions 12 , 289, 311 to 12, 296, 209 (6899 nucleotides in size; Kent et al. 2002). B) A heat map depicting the methylation intensity (in B-values) of specific CPG dinucleotides across EDN1 in HRECs following $48 \mathrm{~h}$ of NG or HG culture. The Illumina methylation array indicates that a B-value of 1 suggests complete methylation at the interrogated site, while a value of 0 indicates no methylation. C) A bar graph categorizing the methylation intensities of probes based on gene region. The proximal promoter and $5^{\prime}$ UTR/first exon regions demonstrated CpG hypomethylation following HG treatment (data expressed as average B-value per region; $N=3$ independent samples for each NG or HG group). * denotes a significant reduction in methylation following HG treatment.

Analyzing the EDN1 regions, there were four probes for TSS1500, five probes for TSS200, one probe for 5' UTR, one probe for gene body, and one probe for 3' UTR. According to our array readout, nearly $58 \%$ of $\mathrm{CpG}$ sites were hypomethylated following $48 \mathrm{~h}$ of $\mathrm{HG}$ treatment (Fig. 1B). In addition, a substantial number of these hypomethylated $\mathrm{CpG}$ sites were predominantly located in the TSS200 region of EDN1, while a hypomethylated CpG site was also detected in both TSS1500 and 5' UTR regions (Fig. 1C). Despite the overall methylation intensities between the $\mathrm{NG}$ and $\mathrm{HG}$ groups being low for each gene region $(0 \leq \beta$-value $\geq 0.119$; a value of 0 indicates no methylation, while a value of 1 indicates maximal methylation), the percentage of methylation further reduced at $\mathrm{CpG}$ sites in the proximal promoter and promoter regions of EDN1 following high glucose treatment.
Global inhibition of DNA methyltransferases increases ET-1 expression

To further investigate the impact of DNA methylation on EDN1 regulation, we administered a DNA demethylating agent in HRECs prior to glucose treatment. Following the global inhibition of DNA methyltransferases, we subsequently analyzed ET-1 mRNA (the main product of EDN1 transcription) and protein expressions using RT-qPCR and ELISA, respectively. Interestingly, 5'-aza-dC administration significantly augmented both ET-1 RNA and protein expressions in NG and HG-treated HRECs compared to controls (Figs 2A and 2C). Among the groups analyzed, HRECs that were cultured with $\mathrm{NG}$ and 5'-aza-dC demonstrated comparable ET-1 RNA and protein expression levels to HRECs cultured solely in HG-suggesting the importance of DNA methyltransferases 
in governing the transcriptional activity of EDN1.

Collectively, both of our DNA methylation experiments indicate that hyperglycemic environments can greatly impact DNA methylation patterns in EDN1 and its transcriptional products, which can ultimately govern translation of the final protein products.

\section{Histone methylation is important in ET-1 regulation}

Following demonstration of DNA methylation's impact on ET-1, we investigated the role of histone methylation on ET-1 mRNA and protein expressions. Therefore, prior to glucose treatment, we subjected HRECs to a global histone methylation inhibitor and subsequently analyzed ET-1 mRNA and protein levels. Similar to the trends observed in our 5'-aza-dC experiment, DZNep pre-treatment significantly upregulated both ET-1 RNA and protein expressions in NG and HG-treated HRECs (Figs 2B and 2D). Following pharmacologic inhibition of histone methylation, the sharp induction of ET-1 expressions in both NG and HG-treated HRECs alludes to the regulatory capabilities of histone methyltransferases on ET-1 transcription and translation.

\section{IncRNAs regulate ET-1 expression in hyperglycemia}

Compared to scrambled controls, approximately $70 \%, 75 \%$, and $90 \%$ knockdown activity was observed in HRECs following siANRIL, siMALAT1, and siZFAS1 treatments, respectively (data not shown). Interestingly, the siRNA-directed inhibition of ANRIL, MALAT1, or ZFAS1 also significantly reduced ET-1 mRNA in HG-treated HRECs (Fig. 3). More specifically, when compared to scrambled controls, ANRIL knockdown contributed to the greatest reduction of ET-1 expression in HG-treated HRECs ( 75\%; Fig. 3A); while, siMALAT1 (Fig. 3B) and siZFAS1 (Fig. 3C) demonstrated comparable levels of ET-1 mRNA reduction ( $\sim 37-42 \%)$ in HG-treated HRECs. Taken together, the findings observed from our knockdown experiments suggest that pathogenetic lncRNAs can also influence glucose-induced upregulation of ET-1 in microvascular endothelial cells (ECs).
A)

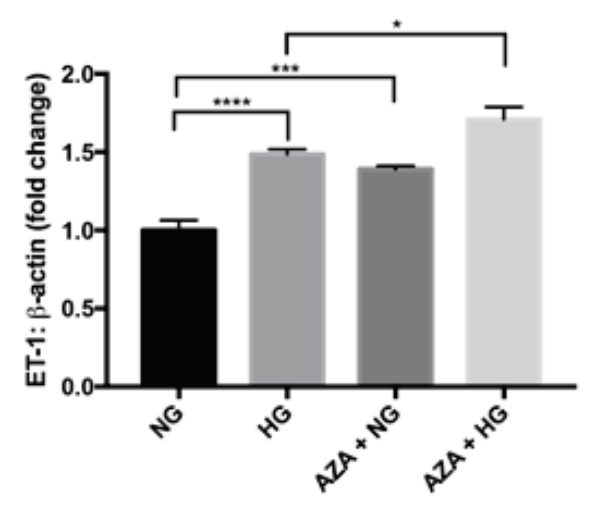

C)

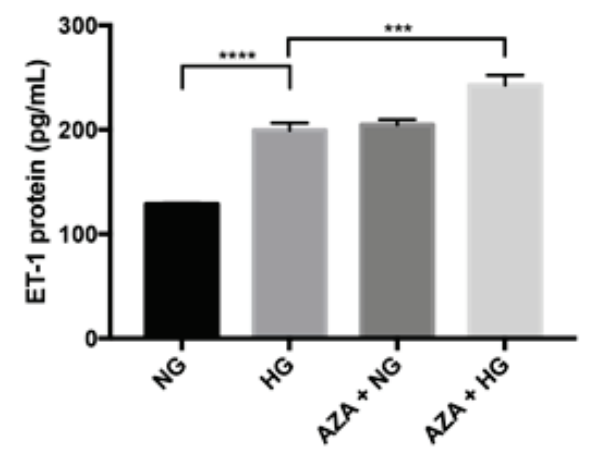

B)

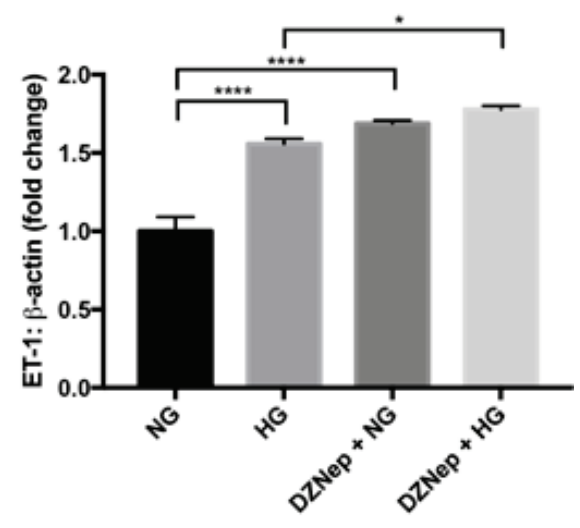

D)

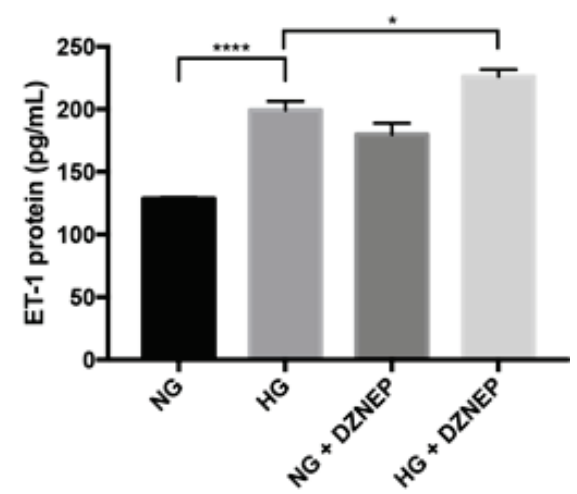

Fig. 2. Global inhibition of DNA or histone methylation significantly increases ET-1 expressions in HRECs. RT-qPCR findings indicate that A) 5-aza-dC treatment increases ET-1 expressions in NG or HG-treated HRECs, B) DZNep treatments also showed similar patterns of ET-1 upregulation (data expressed as a ratio to $\beta$-actin (mean $\pm \mathrm{SEM}$ ); normalized to NG; $* P<0.05, * * * P<0.001$, and $* * * * \mathrm{P}<0.0001$ compared to NG or HG; and $N=6$ from three independent experiments and performed in triplicates). C, D) ELISAs for ET-1 demonstrated elevated ET-1 protein levels (expressed as $\mathrm{pg} / \mathrm{ml}$ ) following 5-aza-dC or DZNep treatment $(* P<0.05, * * * P<0.001$, and $* * * * P<0.0001$ compared to NG or $\mathrm{HG}$; and $N=6$ for each group). 
A)

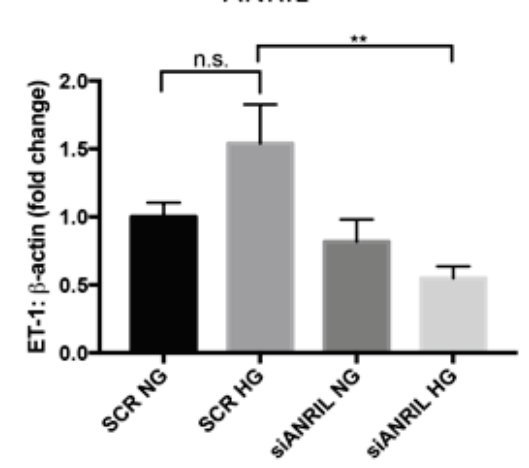

B)

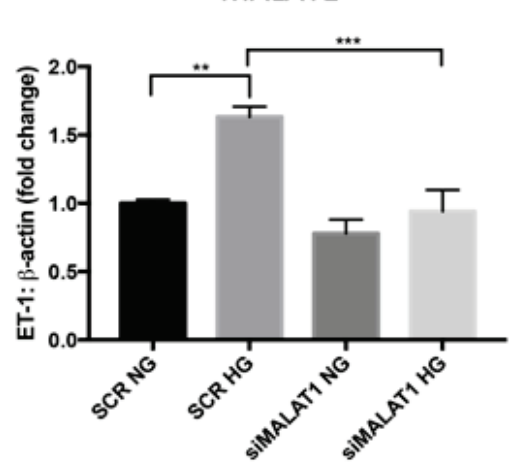

C)

ZFAS1

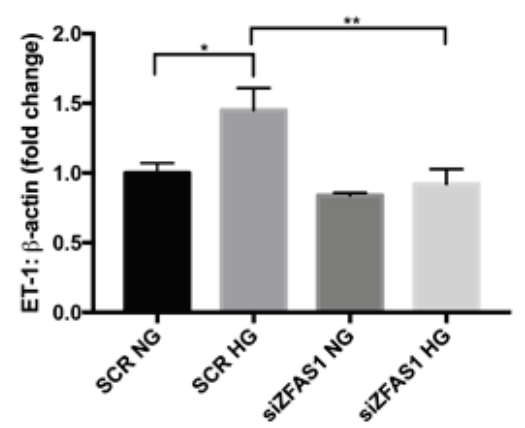

Fig. 3. ANRIL, MALAT1, and ZFAS1 regulate glucose-induced production of ET-1 mRNA in vitro. RT-qPCR analyses indicating that A) SIANRIL, B) siMALAT1, and C) siZFAS1 caused significant reductions in the glucose-induced upregulation of ET-1 transcripts (data expressed as a ratio to $\beta$-actin (mean \pm SEM); normalized to SCR NG; $* P<0.05, * * P<0.01, * * * P<0.001$, and n.s., not significant compared to SCR NG or SCR HG; and $N=6$ from three independent experiments and performed in triplicates).

\section{Discussion}

When a chronic hyperglycemic environment jeopardizes the integrity of the endothelium, the risk of advancement to severe diabetic microvascular complications dramatically heightens. ECs are one of the earliest cells to undergo dysfunction in diabetes, contributing to disease progression (Hadi and Suwaidi 2007). During the development of endothelial dysfunction, one well-documented characteristic is the heightened production and activity of ET-1 (Iglarz and Clozel 2007, Schneider et al. 2002).

Located on chromosome 6, the human EDN1 generates a 2.8-kb transcript that encodes for the pre-proET-1 protein (Yanagisawa et al. 1988). Once pre-proET-1 is generated, the 212-amino-acid protein then undergoes a series of proteolytic modifications to ultimately produce the mature ET-1 peptide (Yanagisawa et al. 1988). Although post-translational mechanisms are moderating ET-1 processing, large bodies of scientific reports indicate that ET-1 bioavailability is mainly directed by transcriptional regulation (Chakrabarti et al. 1998, Chen et al. 2010, Inoue et al. 1989, Stow et al. 2009, Oliver et al. 1991). In fact, the 6.8-kb spanning mammalian EDN1 can be transcriptionally governed by a plethora of transcription factors, which can include hypoxia inducible factor-1, NF- $\kappa$ B, activator protein-1, GATA-2, and c-Myc (Kawana et al. 1995, Quehenberger et al. 2000, Shichiri et al. 1997, Yamashita et al. 2001). Despite the well-documented literature on the various stimuli driving EDN1 transcription, recognizing and incorporating novel regulatory mechanisms, such as epigenetic regulation, into the current ET-1 signaling paradigm will allow for improved understanding and exploration of innovative therapeutics.

Technological advancements in next-generation sequencing have allowed for the identification of genome-wide DNA methylation patterns, which have critical implications in disease pathogenesis. Based on our DNA methylation array, we were able to develop significant insights into the glucose-induced methylation patterns present in the regulatory regions of EDN1. Specifically, in HRECs, transient high glucose exposure sufficiently evoked hypomethylation in the proximal promoter and 5 ' UTR/first exon regions of EDN1. In association, HG-treated HRECs also demonstrated a significant upregulation of ET-1 mRNA and protein at the 48-h mark. Our findings, together with previous 
reports by Han et al. (2011) and Feinberg and Vogelstein (1983), highlight the importance of reduced methylation at $\mathrm{CpG}$ dinucleotides in the regulatory regions of actively transcribed genes. In fact, hypomethylated $\mathrm{CpG}$ sites in the promoter regions of an active gene provide a greater degree of accessibility for transcription factors, which will then facilitate transcription (Deaton and Bird 2011, Eckhardt et al. 2006). Moreover, Vallender and Lahn (2006) have previously indicated that hypermethylation of $\mathrm{CpG}$ regions near intron 1 in $E d n 1$ blocks the binding site for the $\mathrm{Sp} 1$ transcription factor, which may contribute to $E d n 1$ silencing in mice fibroblasts (Vallender and Lahn 2006).

Despite the presence of differential $\mathrm{CpG}$ methylation patterns across EDN1 between NG and HG-treated HRECs, the administration of a global DNA demethylating agent resulted in significant inductions of ET-1 mRNA and protein in both HREC groups. This data is in keeping with past reports in which similar trends for ET-1 expressions were observed in fibroblasts (Vallender and Lahn 2006) and 1833-bone metastatic cells (Matteucci et al. 2013) following the complete inhibition of DNA methylation. These findings, along with our study, suggest that DNA methyltransferases have a critical role in regulating the transcriptional status of $E D N 1$ and that pathological stimuli may be capable of altering the DNA methylating properties of these enzymes in the $E D N 1$ region.

In the context of histone modifications, globally inhibiting histone methylation provoked significant elevations of ET-1 mRNA and protein in HRECs cultured with NG and HG. The profound influence of histone methylation on EDN1 transcriptional activity has been previously documented in renal epithelial cells, where aldosterone treatment induced specific histone conformation changes that maintained active transcription in the EDN1 promoter (Stow et al. 2009). Although chronic hyperglycemic environments can facilitate chromatin remodeling by histone methylation, histone acetylation can also significantly contribute to chromatin state dynamics. Former studies from our laboratory have indicated that the upregulation of histone acetyltransferase p300 can cause increased EDN1 promoter activity (Chen et al. 2009), reduced expression of the class II histone deacetylase SIRT1 (Mortuza et al. 2015), and increased expressions of ET-1 transcripts, extracellular matrix (ECM) proteins and vasoactive factors (Chen et al. 2009, Feng et al. 2011). Collectively, our past and current findings demonstrate that pathologies like diabetes can cause unique alterations in histone modification patterns, which may ultimately enhance EDN1 transcriptional activity and contribute to elevated ET-1 protein levels.

With IncRNAs greatly influencing the epigenetic landscape, we knocked down three pathogenetic glucoseinduced lncRNAs in HRECs in order to determine the impact of IncRNAs on ET-1 transcript expression. Following siRNA-mediated knockdown of ANRIL, MALAT1, and ZFAS1, significant reductions in ET-1 mRNA levels were observed. These findings are consistent with the notion that lncRNAs can alter mRNA transcription by controlling chromatin structure, transcription factors, nuclear organization, or specific protein complexes (Kugel and Goodrich 2013). To further support these notions, we have previously shown that ANRIL can regulate VEGF (vascular endothelial growth factor) through its direct interactions with $\mathrm{p} 300$ and PRC2 (polycomb repressive complex 2; a histone methyltransferase) in HRECs (Thomas et al. 2017). Such a mechanistic study is warranted for ET-1, so that the direct relationship between epigenetic mediator proteins and EDNI can be confirmed in a diabetic context. Moreover, we have previously demonstrated the importance of microRNAs (miRNAs) 1 and 320 in ET-1 regulation (Feng et al. 2014, Feng and Chakrabarti 2012), miR-200b in mediating PRC2 and p300 activities (Ruiz et al. 2015, McArthur et al. 2011), and miR-146a impacting ECM proteins (Feng et al. 2011), inflammatory cytokines (Chen et al. 2017), and endothelial-to-mesenchymal transitioning (Cao et al. 2014). Therefore, based on our past and current findings, we collated the pertinent information and developed an epigenetic paradigm for ET-1 regulation (Fig. 4).

Since we have reported alterations in ET-1 expression following global inhibition of histone and DNA methylation, and lncRNA knockdown, it is important to note that these changes may reflect a more widespread phenomenon extending to other genes. As well, the alterations observed in our experiments are based on a single time point, which only provides a snapshot of the epigenetic phenomena on ET-1 regulation. We understand that these are limitations of our study and additional work is warranted to look into additional time points and identify other key genes implicated in this intricate network. Future experiments should also investigate the impact of epigenetic mechanisms on ET receptors, as these receptors play an important role in eliciting the physiological responses of ET-1 upon binding. 


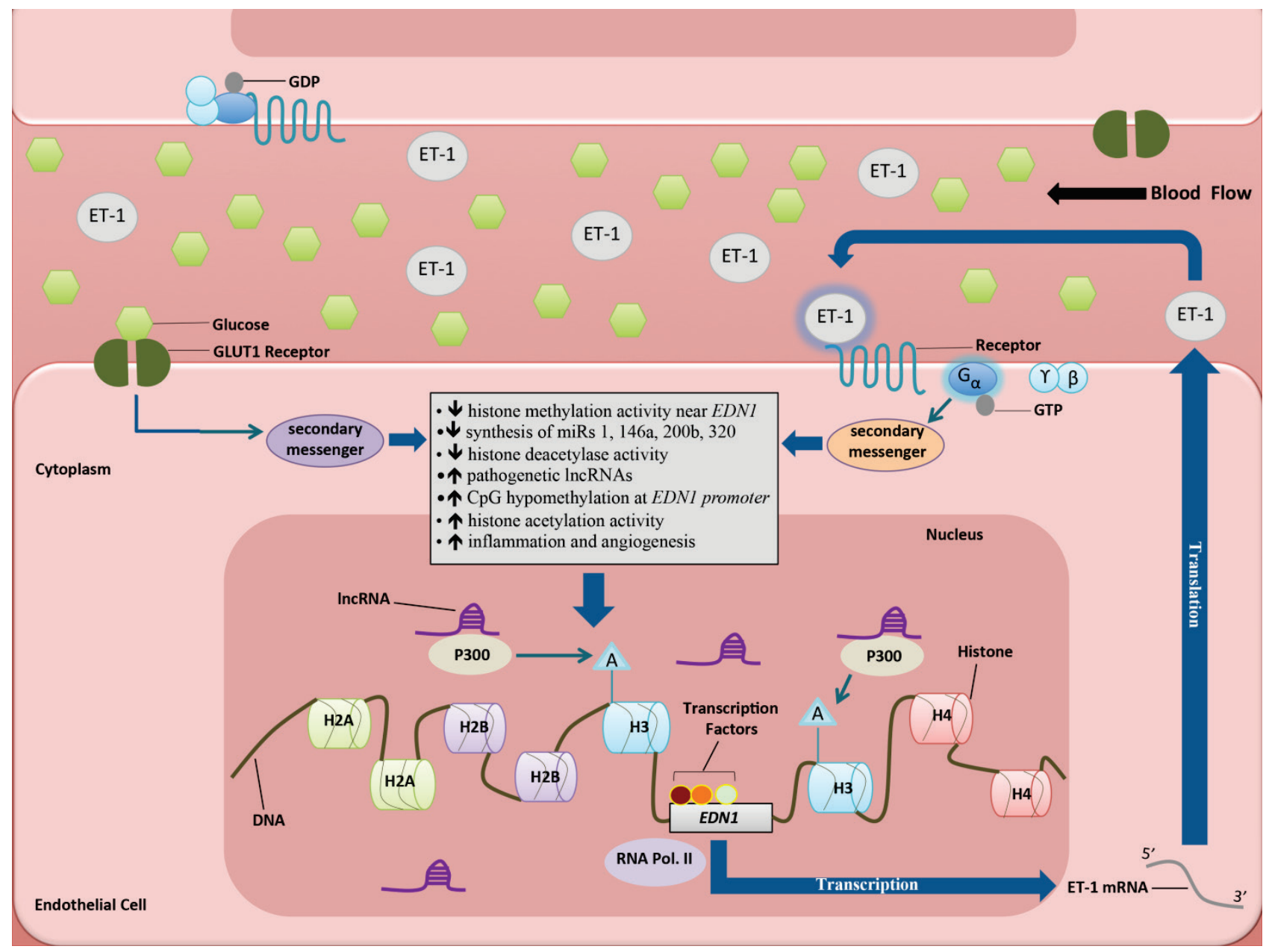

Fig. 4. An illustration depicting the proposed epigenetic paradigm underlying ET-1 regulation in microvascular endothelial cells in diabetes. Based on our previous and current findings, we propose this epigenetic model in which histone modifications, DNA methylation, miRNAs, and IncRNAs can possibly influence EDN1 transcriptional activity and ultimately ET-1 RNA and protein levels. GDP, guanosine diphosphate; GTP, guanosine triphosphate; IncRNA, long non-coding RNA; miRs, microRNAs; p300, histone acetyltransferase; H2A-H4, histones; RNA Pol. II, RNA polymerase II; and A, acetylation.

Incorporation of the emerging regulatory mechanisms into the previously understood paradigm of ET-1 signaling becomes imperative in the development of novel ET-1-based therapeutics. Although the findings from our study shed novel insights into the epigenetic influence on ET-1 regulation, further mechanistic studies are necessary to elucidate the direct relationship between epigenetic mediator proteins and ET-1 in the diabetes context.

\section{Conflict of Interest}

The authors have declared no conflicts of interest. Manuscript comments: The illustration in Figure 4 was drawn by Saumik Biswas and Subrata Chakrabarti. We provide permission to Physiological Research to publish our illustration in all formats (i.e. print and digital).

\section{Acknowledgements}

This research was supported by the Heart and Stroke Foundation of Canada.

\section{References}

ALDER VA, SU EN, YU DY, CRINGLE SJ, YU PK: Diabetic retinopathy: early functional changes. Clin Exp Pharmacol Physiol 24: 785-788, 1997. doi:10.1111/j.1440-1681.1997.tb02133.x.

BISWAS S, CHAKRABARTI S: Pathogenetic mechanisms in diabetic retinopathy: from molecules to cells to tissues. In: Mechanisms of Vascular Defects in Diabetes Mellitus. KARTHA CC, RAMACHANDRAN S, PILLAI RM (eds), Springer, 2017, p. 209-247. doi:10.1007/978-3-319-60324-7_9. 
CAO Y, FENG B, CHEN S, CHU Y, CHAKRABARTI S: Mechanisms of endothelial to mesenchymal transition in the retina in diabetes. Investig Ophthalmol Vis Sci 55: 7321-7331, 2014. doi:10.1167/iovs.14-15167.

CHAKRABARTI S, GAN XT, MERRY A, KARMAZYN M, SIMA AA: Augmented retinal endothelin-1, endothelin-3, endothelinA and endothelinB gene expression in chronic diabetes. Curr Eye Res 17: 301-307, 1998. doi:10.1076/ceyr.17.3.301.5216.

CHAWLA A, CHAWLA R, JAGGI S: Microvasular and macrovascular complications in diabetes mellitus: distinct or continuum? Indian J Endocrinol Metab 20: 546, 2016. doi:10.4103/2230-8210.183480.

CHEN S, APOSTOLOVA MD, CHERIAN MG, CHAKRABARTI S: Interaction of endothelin-1 with vasoactive factors in mediating glucose-induced increased permeability in endothelial cells. Lab Invest 80: 1311-1321, 2000. doi:10.1038/labinvest.3780139.

CHEN S, FENG B, GEORGE B, CHAKRABARTI R, CHEN M, CHAKRABARTI S: Transcriptional coactivator p300 regulates glucose-induced gene expression in endothelial cells. Am J Physiol Endocrinol Metab 298: E127E137, 2010. doi:10.1152/ajpendo.00432.2009.

CHEN S, FENG B, THOMAS AA, CHAKRABARTI S: MiR-146a regulates glucose induced upregulation of inflammatory cytokines extracellular matrix proteins in the retina and kidney in diabetes. PLoS One 12: 29752984, 2017. doi:10.1371/journal.pone.0173918.

DEATON A, BIRD A: CpG islands and the regulation of transcription. Genes Dev 25: 1010-1022, 2011. doi:10.1101/gad.2037511.1010.

ECKHARDT F, LEWIN J, CORTESE R, RAKYAN VK, ATTWOOD J, BURGER M, BURTON J, COX TV, DAVIES R, DOWN TA, HAEFLIGER C, HORTON R, HOWE K, JACKSON DK, KUNDE J, KOENIG C, LIDDLE J, NIBLETT D, OTTO T, PETTETT R, SEEMANN S, THOMPSON C, WEST T, ROGERS J, OLEK A, BERLIN K, BECK S: DNA methylation profiling of human chromosomes 6, 20 and 22. Nat Genet 38: 1378-1385, 2006. doi: 10.1038/ng1909.

FEINBERG AP, VOGELSTEIN B: Hypomethylation distinguishes genes of some human cancers from their normal counterparts. Nature 301: 89-92, 1983. doi:10.1038/301089a0.

FENG B, CAO Y, CHEN S, RUIZ M, CHAKRABARTI S: miRNA-1 regulates endothelin-1 in diabetes. Life Sci 98: 18-23, 2014. doi:10.1016/j.lfs.2013.12.199.

FENG B, CHAKRABARTI S: miR-320 regulates glucose-induced gene expression in diabetes. ISRN Endocrinol 2012: 1-6, 2012. doi:10.5402/2012/549875.

FENG B, CHEN S, MCARTHUR K, WU Y, SEN S, DING Q, FELDMAN RD, CHAKRABARTI S: miR-146amediated extracellular matrix protein production in chronic diabetes complications. Diabetes 60: 2975-2984, 2011. doi:10.2337/db11-0478.

FOWLER MJ: Microvascular and macrovascular complications of diabetes. Clin Diabetes 29: 116-122, 2011. doi:10.2337/diaclin.29.3.116.

GARDINER-GARDEN M, FROMMER M: CpG islands in vertebrate genomes. J Mol Biol 196: 261-282, 1987. doi:10.1016/0022-2836(87)90689-9.

HADI HA, SUWAIDI JA: Endothelial dysfunction in diabetes mellitus. Vasc Health Risk Manag 3: 853-876, 2007. doi:10.1038/sj.bjp.0703393.

HAN H, CORTEZ CC, YANG X, NICHOLS PW, JONES PA, LIANG G: DNA methylation directly silences genes with non-CpG island promoters and establishes a nucleosome occupied promoter. Hum Mol Genet 20: 42994310, 2011. doi:10.1093/hmg/ddr356.

INOUE A, YANAGISAWA M, TAKUWA Y, MITSUI Y, KOBAYASHI M, MASAKI T: The human preproendothelin-1 gene. Complete nucleotide sequence and regulation of expression. J Biol Chem 264: 14954-14959, 1989.

IGLARZ M, CLOZEL M: Mechanisms of ET-1-induced endothelial dysfunction. J Cardiovasc Pharmacol 50: 621-628, 2007. doi:10.1097/FJC.0b013e31813c6cc3.

IDF: Diabetes Atlas. International Diabetes Federation, 2015. doi:10.1289/image.ehp.v119.i03.

KAWANA M, LEE ME, QUERTERMOUS EE, QUERTERMOUS T: Cooperative interaction of GATA-2 and AP1 regulates transcription of the endothelin-1 gene. Mol Cell Biol 15: 4225-4231, 1995. doi:10.1128/MCB.15.8.4225. 
KENT WJ, SUGNET CW, FUREY TS, ROSKIN KM, PRINGLE TH, ZAHLER AM, HAUSSLER D: The human genome browser at UCSC. Genome Res 12: 996-1006, 2002. doi: 10.1101/gr.229102.

KHAN ZA, CUKIERNIK M, GONDER JR, CHAKRABARTI S: Oncofetal fibronectin in diabetic retinopathy. Investig Ophthalmol Vis Sci 45: 287-295, 2004. doi:10.1167/iovs.03-0540.

KOHAN DE, POLLOCK DM: Endothelin antagonists for diabetic and non-diabetic chronic kidney disease. Br J Clin Pharmacol 76: 573-579, 2013. doi:10.1111/bcp.12064.

KUGEL JF, GOODRICH JA: The regulation of mammalian mRNA transcription by lncRNAs: recent discoveries and current concepts. Epigenomics 5: 95-102, 2013. doi:10.2217/epi.12.69.

MATTEUCCI E, MARONI P, BENDINELLI P, LOCATELLI A, DESIDERIO MA: Epigenetic control of endothelin-1 axis affects invasiveness of breast carcinoma cells with bone tropism. Exp Cell Res 319: 1865-1874, 2013. doi:10.1016/j.yexcr.2013.04.022.

MCARTHUR K, FENG B, WU Y, CHEN S, CHAKRABARTI S: MicroRNA-200b regulates vascular endothelial growth factor-mediated alterations in diabetic retinopathy. Diabetes 60: 1314-1323, 2011. doi:10.2337/db101557.

MORTUZA R, FENG B, CHAKRABARTI S: SIRT1 reduction causes renal and retinal injury in diabetes through endothelin 1 and transforming growth factor $\beta 1 . J$ Cell Mol Med 19: 1857-1867, 2015. doi:10.1111/jcmm.12557.

OLIVER FJ, DE LA RUBIA G, FEENER EP, LEE ME, LOEKEN MR, SHIBA T, QUERTERMOUS T, KING GL: Stimulation of endothelin-1 gene expression by insulin in endothelial cells. J Biol Chem 266: 23251-23256, 1991.

PUTHANVEETIL P, CHEN S, FENG B, GAUTAM A, CHAKRABARTI S: Long non-coding RNA MALAT1 regulates hyperglycaemia induced inflammatory process in the endothelial cells. J Cell Mol Med 19: 1418-1425, 2015. doi:10.1111/jcmm.12576.

QUEHENBERGER P, BIERHAUS A, FASCHING P, MUELLNER C, KLEVESATH M, HONG M, STIER G, SATTLER M, SCHLEICHER E, SPEISER W, NAWROTH PP: Endothelin 1 transcription is controlled by nuclear factor-kappaB in AGE-stimulated cultured endothelial cells. Diabetes 49: 1561-1570, 2000. doi:10.2337/diabetes.49.9.1561.

REMUZZI G, PERICO N, BENIGNI A: New therapeutics that antagonize endothelin: promises and frustrations. Nat Rev Drug Discov 1: 986-1001, 2002. doi:10.1038/nrd962.

RUIZ MA, FENG B, CHAKRABARTI S: Polycomb repressive complex 2 regulates MiR-200b in retinal endothelial cells: Potential relevance in diabetic retinopathy. PLoS One 10: 1-21, 2015. doi:10.1371/journal.pone.0123987.

ROBERTO T, RITA BA, PRATTICHIZZO F, LA SALA L, DE NIGRIS V, CERIELLO A: The "metabolic memory" theory and the early treatment of hyperglycemia in prevention of diabetic complications. Nutrients 9: 1-9, 2017. doi:10.3390/nu9050437.

SASSER JM, SULLIVAN JC, HOBBS JL, YAMAMOTO T, POLLOCK DM, CARMINES PK, POLLOCK JS: Endothelin A receptor blockade reduces diabetic renal injury via an anti-inflammatory mechanism. $J$ Am Soc Nephrol 18: 143-154, 2007. doi:10.1681/ASN.2006030208.

SCHNEIDER JG, TILLY N, HIERL T, SOMMER U, HAMANN A, DUGI K, LEIDIG-BRUCKNER G, KASPERK C: Elevated plasma endothelin-1 levels in diabetes mellitus. Am J Hypertens 15: 967-972, 2002. doi:10.1016/S0895-7061(02)03060-1.

SHICHIRI M, ADACHI S, SEDIVY JM, MARUMO F, HIRATA Y: Biphasic regulation of the preproendothelin-1 gene by c-myc. Endocrinology 138: 4584-4590, 1997. doi:10.1210/en.138.11.4584.

STOW LR, GUMZ ML, LYNCH IJ, GREENLEE MM, RUDIN A, CAIN BD, WINGO CS: Aldosterone modulates steroid receptor binding to the endothelin-1 gene (edn1). J Biol Chem 284: 30087-30096, 2009. doi:10.1074/jbc.M109.030718.

TAKAHASHI K, GHATEI MA, LAM HC, O'HALLORAN DJ, BLOOM SR: Elevated plasma endothelin in patients with diabetes mellitus. Diabetologia 33: 306-310, 1990. doi:10.1007/BF00403325.

THOMAS AA, FENG B, CHAKRABARTI S: ANRIL: a regulator of VEGF in diabetic retinopathy. Investig Ophthalmol Vis Sci 58: 470-480, 2017. doi:10.1167/iovs.16-20569. 
VALLENDER TW, LAHN BT: Localized methylation in the key regulator gene endothelin-1 is associated with cell type-specific transcriptional silencing. FEBS Lett 580: 4560-4566, 2006. doi:10.1016/j.febslet.2006.07.017.

WEINHOLD B: Epigenetics: the science of change. Environ Heal Perspect 114: A160-A167, 2006. doi:10.1289/ehp.114-a160.

XIE M, TIAN J, LUO Y, WEI L, LIN S, TANG S: Effects of 5-aza-2'-deoxycytidine and trichostatin A on high glucose- and interleukin-1 $\beta$-induced secretory mediators from human retinal endothelial cells and retinal pigment epithelial cells. Mol Vis 20: 1411-1421, 2014.

YAMASHITA K, DISCHER DJ, HU J, BISHOPRIC NH, WEBSTER KA: Molecular regulation of the endothelin-1 gene by hypoxia. Contributions of hypoxia-inducible factor-1, activator protein-1, GATA-2, and p300/CBP. J Biol Chem 276: 12645-12653, 2001. doi:10.1074/jbc.M011344200.

YANAGISAWA M, KURIHARA H, KIMURA S, TOMOBE Y, KOBAYASHI M, MITSUI Y, YAZAKI Y, GOTO K, MASAKI T: A novel potent vasoconstrictor peptide produced by vascular endothelial cells. Nature 332: 411-415, 1988. doi:10.1038/332411a0. 